\title{
First flight model optical performance test on the Flexible Combined Imager telescope assembly of Meteosat Third Generation
}

S. Fray, J. Hofmann, Alessandro Boni, T. Deuschle, C. Küchel, et al.

S. Fray, J. Hofmann, Alessandro Boni, T. Deuschle, C. Küchel, U. Förster, J. Plumhof, M. Gerstmeier, G. Bambina, G. Petitjean, J. Ouaknine, J. Durand, P. Hallibert, "First flight model optical performance test on the Flexible Combined Imager telescope assembly of Meteosat Third Generation," Proc. SPIE 11852, International Conference on Space Optics - ICSO 2020, 1185212 (11 June 2021); doi: 10.1117/12.2599222

SPIE Event: International Conference on Space Optics - ICSO 2021, 2021, Online Only 


\section{International Conference on Space Optics-ICSO 2020}

Virtual Conference

30 March-2 April 2021

Edited by Bruno Cugny, Zoran Sodnik, and Nikos Karafolas
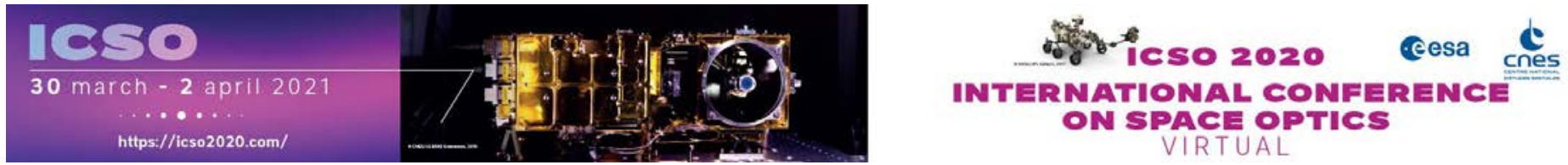

First flight model optical performance test on the Flexible Combined Imager telescope assembly of Meteosat Third Generation

\section{Cesa issopocesalings lecnes}




\title{
First Flight model Optical performance test on the Flexible Combined Imager Telescope Assembly of Meteosat Third Generation
}

\author{
S. Fray ${ }^{\mathrm{a}}$, J. Hofmann ${ }^{\mathrm{a}}$ A. Boni*a, T. Deuschle ${ }^{\mathrm{a}}$, C. Küchel ${ }^{\mathrm{a}}$, U. Förster ${ }^{\mathrm{a}}$, J. Plumhof ${ }^{\mathrm{a}}$ M. Gerstmeier ${ }^{\mathrm{a}}$, \\ G. Bambina ${ }^{\mathrm{a}}$, G. Petitjean ${ }^{\mathrm{b}}$, J. Ouaknine ${ }^{\mathrm{c}}$, J. Durand ${ }^{\mathrm{b}}$, P. Hallibert ${ }^{\mathrm{b}}$ \\ ${ }^{\mathrm{a} O H B}$ system AG, Manfred Fuchs Straße 1, D-82234 Weßling / Oberpfaffenhofen, Germany \\ ${ }^{b}$ European Space Agency, ESTEC, Keplerlaan 1, NL-2200 AG Noordwijk \\ ${ }^{\mathrm{c}}$ Thales Alenia Space, 5, allée des gabians, BP 99 - 06156 Cannes - France
}

\begin{abstract}
The Flexible Combined Imager (FCI), main instrument of the Meteosat Third Generation Imager (MTG-I) satellite, will image the on-Earth instrument swath with high image quality and highly stable line of sight. It is composed by a Three Mirror Anastigmatic (TMA) telescope assembly, followed by a Spectral Separation \& Detection Assembly (SSDA) which has the function to separate the different spectral channels and focus them onto the detector. The Telescope assembly, developed by OHB System AG in Oberpfaffenhofen, will be the subject of this article, presenting first its optical design concept and its main characteristics. Thereafter, the FCI Telescope Assembly (FCI-TA) alignment will be presented, followed by the full test campaign accomplished to characterize the full optical performance of the FCI-TA. The telescope optical design is based on an off-axis field TMA telescope. It is composed by three aspheric off-axis mirrors, having a focal length of $1650 \mathrm{~mm}$, an F/\# of 5.5 and an entrance pupil diameter of $302 \mathrm{~mm}$. A fourth, folding, mirror deflects the beam onto the TA focal plane, while in front of the first TMA mirror there is a Scan mirror, in order to let the telescope scan the whole Earth disc. In mid-2019 OHB ended the alignment of this first flight model of FCI-TA. We will show in this article the good results in terms of optical performance obtained from that alignment with a worst WFE of $63 \mathrm{~nm}$ and a LOS error of 7.8 arcsec.
\end{abstract}

Keywords: Imager, climate, optical design, optical tests, alignment

\section{INTRODUCTION}

The Flexible Combined Imager (FCI) is the main instrument of the MTG-I satellite, which will be part of the Meteosat Third Generation (MTG) constellation, the future European Operational system that will replace the Second Generation (MSG) at the end of its operational life. The challenge of the MTG constellation will be to continue and improve weather forecasting capabilities and other services for Europe, such as improved air quality, UV-radiation warning, as well as climate and atmospheric chemistry monitoring. The space components of the MTG system will be made up of a multiple satellites constellation. In order to span the operational lifetime of the program over 20 years, there will be four satellites dedicated to support the Imagery missions (MTG-I), and two satellites to support the sounding missions (MTG-S). All satellites will be operating at geostationary orbit.

The Flexible Combined Imager (FCI) provides the imagery mission composed of the High Resolution Imagery (HRFI) and the Full Disk High Spectral Imagery (FDHSI). During its operational lifetime of 8.5 years, the instrument will image the on-earth instrument swath with high image quality and a highly stable line-of sight. It can fulfill both the FDHSI by scanning the full earth disc in 16 channels every 10 minutes with a resolution of 1-2 km and the HRFI Mission which will cover a quarter of the earth in 4 channels every 2.5 minutes with a resolution of $0.5-1 \mathrm{~km}$. The instrument is designed to withstand the regular solar intrusions during its operational phase to ensure a minimum of outages. It is envisaged to allow uninterrupted observation even when the instrument is subjected to solar intrusions inside its cavity. Both visible (VIS) and InfraRed (IR) channels are routinely calibrated by a dedicated calibration unit.

*alessandro.boni@ohb.de; phone +49 8153-4002-677; ohb.de 
OHB is responsible for the design and integration of the FCI Instrument. It is composed by a Three Mirror Anastigmatic (TMA) Telescope Assembly (TA), mounted onto an Optical bench (OBA), followed by a Spectral Separation \& Detection Assembly (SSDA), which separates the beam into several spectral channels and focuses them onto the detectors. The latter is subject of another paper covered by the Instrument prime, TAS [1]. A Scan mirror Assembly (SCA) placed in front of the primary mirror of the TMA provides the TA with the capability of imaging the Earth disc in separate and successive swaths connected to each other via U-turn motions. This movement is controlled by its dedicated electronics physically located inside the platform.

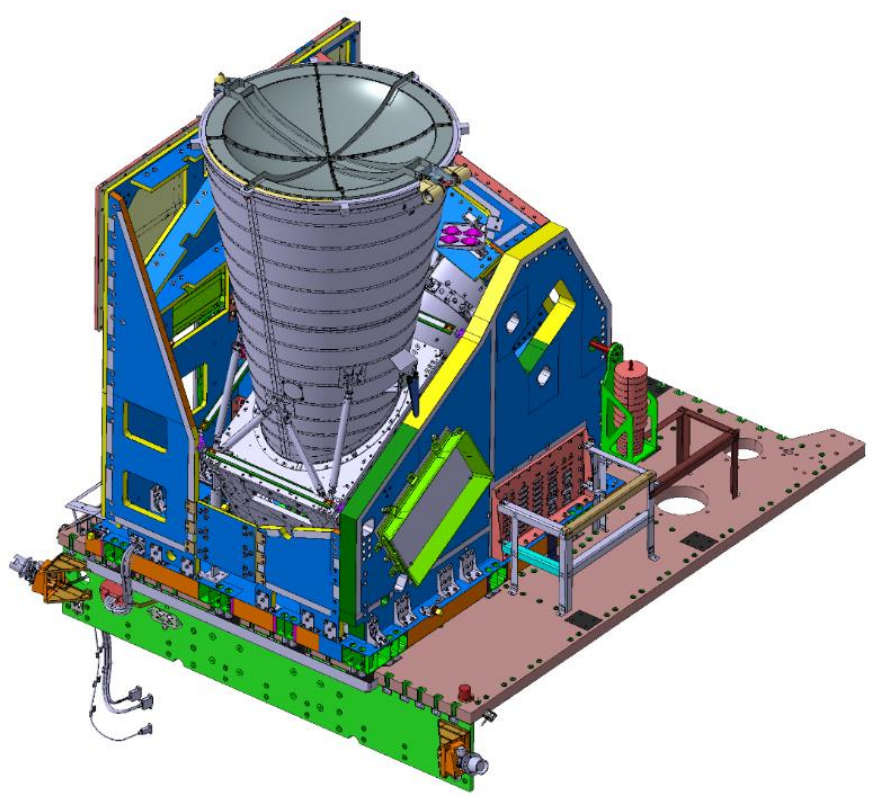

Figure 1 Model of the FCI-TA mounted on its support structure

This paper will begin with a more detailed description of the TA optical design concept and its most important optical performance in design conditions. A subsequent section will describe in detail the alignment concept with its success criteria and the tools used to achieve them. Following we will introduce a section of this paper describing the test campaign carried out at OHB premises on the TA assembly. The paper will then draw some conclusions and future perspectives.

\section{OPTICAL DESIGN CONCEPT}

The optical design of the FCI-TA is based on an off-axis field, off-axis pupil Three Mirror Anastigmat (TMA) telescope type, purely reflective to be compatible with a multi-channel instrument, with a $1.4^{\circ}$ off-axis angle in the nominal configuration. All three mirrors are off-axis, aspheric. Before the first mirror a Scanning mirror is placed, approximately at the Entrance Pupil (EP) position, which scans the overall Earth disc with its mechanism. An additional folding mirror before the focal plane deflect the rays onto the detection assembly, arranged in another plane. All the mirrors are light weighted and made of Zerodur, except the scanning mirror made of $\mathrm{SiC}$, with space-qualified silver protected coatings. A schematic view of the arrangement is shown in the following picture (Figure 2).

The design concept of this telescope includes two additional components: an intermediate focal plane, Field Stop (FS), right after the secondary mirror, whose functionality is to limit straylight into the optical path and Sun intrusion into the instrument, an Aperture Stop (AS), before the folding mirror M4, to set the EP dimension.

A REfocusing Mechanism (REM) is introduced at the level of M2 to ensure the optical quality of the instrument in-flight by compensating effects from gravity and moisture release. 

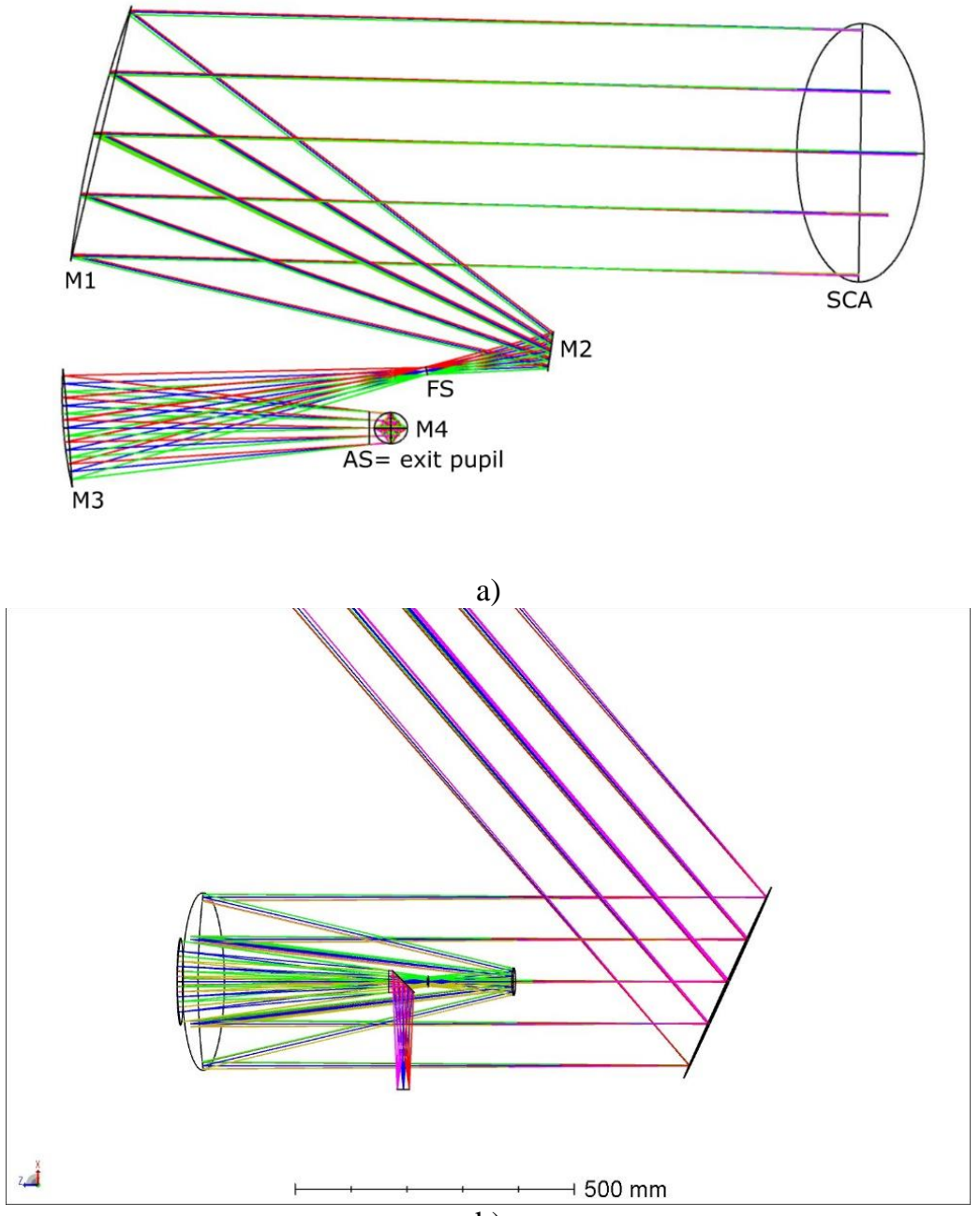

b)

Figure 2 TMA optical scheme: a) top view b) side view

The telescope is designed to cover different fields of view for the different channels described in Table 1, with a separation between VNIR and WVIR. During scans, performed in E/W direction, all spectral channels scan the same ground track just slightly delayed with respect to each other.

Table 1 FCI-TA Field of View. E/W and N/S directions represent direction onto the Earth

\begin{tabular}{|l|l|l|l|l|l|l|}
\hline & & NIR & IR1 & IR2 & IR3 & VIS \\
\hline FOV (+/-) & E/W & 0.2 & 0.37 & 0.37 & 0.37 & 0.2 \\
\hline & N/S & 0.2 & 0.2 & 0.2 & 0.2 & 0.2 \\
\hline
\end{tabular}

During optimization particular care was given on the control of performance during different end-to-end Monte Carlo scenarios of the TA during its life. A slight asymmetry in the common axis of symmetry was introduced, while keeping the off-axis alignment (mirrors vertex lay on the same optical plane). This produced much more stable performance with reduced WFE across the field of view. 


\section{TA ALIGNMENT}

This subsection describes the placement and optical alignment of the FCI-TA mirrors. It discusses the alignment concept including the required tools as well as the alignment results of the first flight models. The logic is based on a concept developed and used by OHB to align the EnMAP instrument.

\subsection{Alignment success criteria}

The FCI-TA alignment requirements are mainly driven by the worst-case imagette Wavefront Error (WFE) and the Line Of Sight (LOS) error. The FCI-TA alignment concept is developed to guarantee a sufficient WFE and LOS error, while minimizing number of alignment loops and thus the time required for the alignment. The alignment concept further respects the interface geometries and the resulting boundary conditions.

The success criterion for the FCI-TA WFE alignment is calculated individually for each model. The value is based on a Zemax-model featuring the measured SFE of every mirror, an alignment budget based on optical tolerancing and a WFE budget for attachment tolerances. The success criteria was approximately $100 \mathrm{~nm}$ (WFE single pass) for the already aligned flight models. The criterion is a contributor for the global WFE budget.

The success criterion for the LOS alignment is strictly connected to the main reference frame of the FCI-TA. The LOS vector shall deviate by less than 50 arcsec from the nominal LOS vector after alignment.

Further success criteria describe the position of the physical aperture stop and field stop. These components are placed to the optical bench and not moved during alignment. The correct placement is verified by budgets supported by CMM measurements. As these components are not actively aligned based on an optical signal, their placement is not in the scope of this paper.

\subsection{FCI-TA hardware overview}

All four mirrors of the FCI-TA placed on an optical bench assembly (OBA). The M1 and M3 mirror are placed directly to the OBA, whereas the M4 mirror is mounted on a substructure, which interfaces with the OBA. The M4 substructure additionally supports the field stop and aperture stop. The M2 mirror is mounted to the REM, which allows M2 movements along the optical axis of M2 in flight, in order to correct defocus. M2 and REM are mounted to the optical bench as an assembly with OBA interface points on the REM part of the assembly. A schematic of the whole assembly is shown in Figure 3. The optical beam path is already presented in Figure 2.

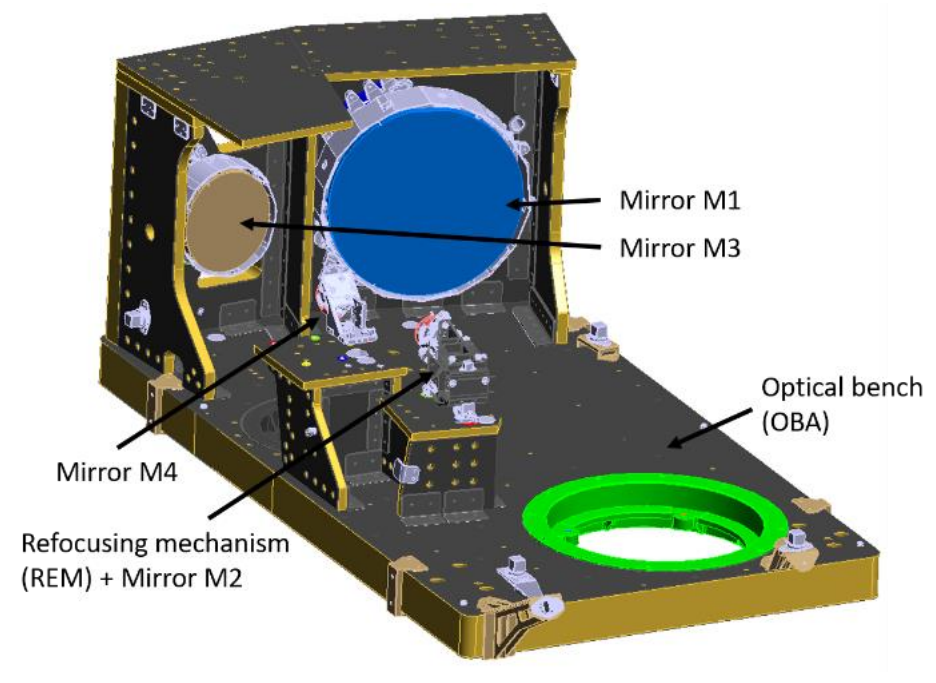

Figure 3 Hardware assembly (OBA and mirrors)

M1 and M3 mirrors interface the OBA on three mounting points (rotated by $120^{\circ}$ with respect to reach other). The mounting interfaces (MFD) are connected to the respective mirror structure by bipods. During all handling operations of the mirrors, a conformer ring is mounted to each mirror, hard fixing the position of the MFDs with respect to each other. The spatial and angular position of the mirrors are fixed by wedged shims between the each MFD and the OBA. Each shim is positioned to the OBA by two pins. The shim compensate flatness tolerances of the OBA interfaces. Therefore, the 
mirror-interfacing plane, given by the three shims for the respective mirror, is always a flat plane. One shim per mirror has an additional pinhole and one has a long hole. The holes overlap with holes in the mirror MFDs and are used to fix the in plane and clocking degrees of freedom of the mirror with respect to the OBA by a pinned connection. The out of plane and tip/tilt degrees of freedom are fixed by the thickness of the shims and their wedge. Figure 4 shows a schematic of the concept.

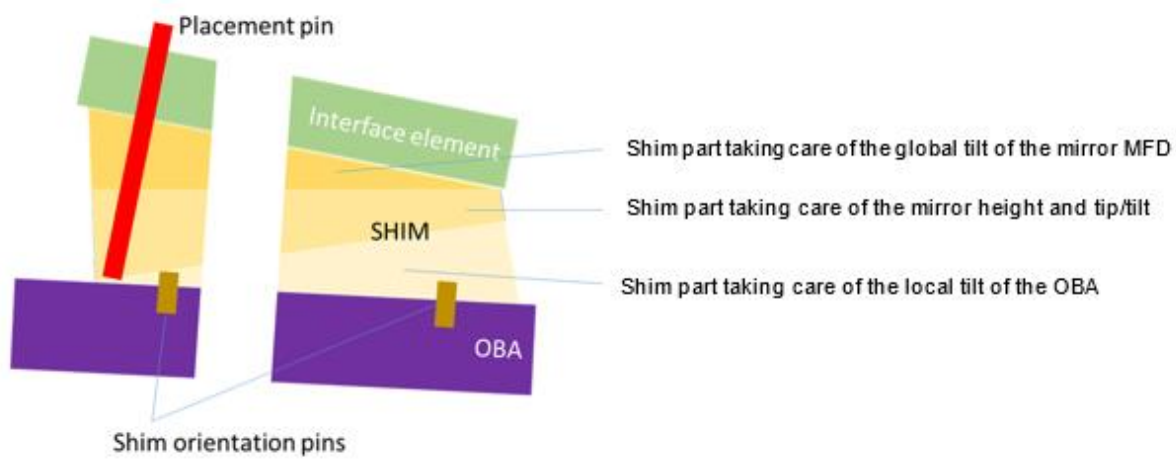

Figure 4 M1 and M3 shim design.

The M4 mirror is fixed to its substructure by three blades. During placement, M4 and substructure are considered as a fixed assembly. The out of plane and tip/tilt position of the assembly is defined by a single wedged shim, fixed to the OBA by two pins (analogously to M1 and M3). The in plane and clocking degrees of freedom are locked differently as for M1 and M3. A push-pull GSE is installed, which can move the M4 assembly on the shim plane. This setup allows a more precise M4 in-plane alignment.

After mounting the M2 to the REM, the M2-REM assembly is also considered to be a fixed assembly. The REM interfaces the OBA on 3 points. On each interface, a stack of a wedged shim and a flat shim is used. The position and orientation of the wedged shims are again fixed to the OBA by pins. The in-plane and clocking degrees of freedom are aligned by a push pull GSE, analogously to M4.

\subsection{Alignment Process}

The placement and alignment of the mirrors on the OBA is mainly controlled by pinned shims or by a combination of shims and an alignment GSE as presented in the previous subsection. The concept is based on a three-step process, in which the second and third step are optional. Either one of the two steps or both steps can be executed. The three steps are placement, alignment and fine-alignment. Figure 5 gives a conceptual overview about the alignment flow.

The alignment already starts with the first placement of the mirrors onto the OBA. This step places the apex of each mirror to a target position with respect to the OBA based on a Zemax simulation, which includes the as built data of the individual mirrors. The correct shims are calculated based on CMM measurements of the OBA and mirror interfaces, whose position is also characterized with respect to the respective mirror apex. The calculation is performed by a so-called interface generation (IFG) software developed by $\mathrm{OHB}$ and generates directly the dimensional information for all shimmanufacturing processes [2]. The accuracy of the placement process is mainly driven by the knowledge of the positon and orientation of individual mirror apex with respect to the mirror to structure interfaces. After each mirror integration, the gravity-introduced deformations of the Optical Bench caused by the weight of the individual mirrors have been compensated using an adaptive push-pull GSE mounted below the OBA and alignment table. The compensation was based on live-measurements of the OBA deformations.

The placement is followed by an analysis based on an optical measurement of the FCI-TA WFE and LOS. The analysis checks, if the placement was successful and alignment process can move on with the next step or, whether the placement needs to be repeated. The calculations are performed by a so-called SOFA software developed by OHB. It calculates the mirror movements required to align the FCI-TA as function of selected degrees of freedom for the movements of each mirror. In case only movements of $\mathrm{M} 2$ and $\mathrm{M} 3$ are required to align the telescope, the placement is successful and the next step of the alignment concept is executed. The alignment step utilizes movements of M2 in all degrees of freedom and 
tip/tilts M3 to align the telescope. This step is even skipped, if the calculations with SOFA show that the final success criteria for the telescope alignment can be reached by movements of only M2.

The required alignment-movements of M2 and M3 are calculated by the SOFA software and are a direct input to the IFG software. The IFG software calculates new sets of wedged shims for M2 and M3 and a delta in-plane position of the M2REM assembly. In order to implement the new shims, M3 and the M2-REM assembly are dismounted and remounted on the new shims. The new in-plane position of M2 is accomplished by delta movements of the M2-REM assembly with the still installed push-pull GSE.

A step is repeated until the criteria for a subsequent step is fulfilled
Placement

Based on characterization

data and Zemax simulation

TA can be aligned by movements of $\mathrm{M} 2$ and $\mathrm{M} 3$

\section{Alignment of M2 \\ and $\mathrm{M} 3$ \\ Based on optical measurements}

TA can be aligned by fine -movements of $\mathrm{M} 2$

WFE and LOS error fulfill the alignment success criteria

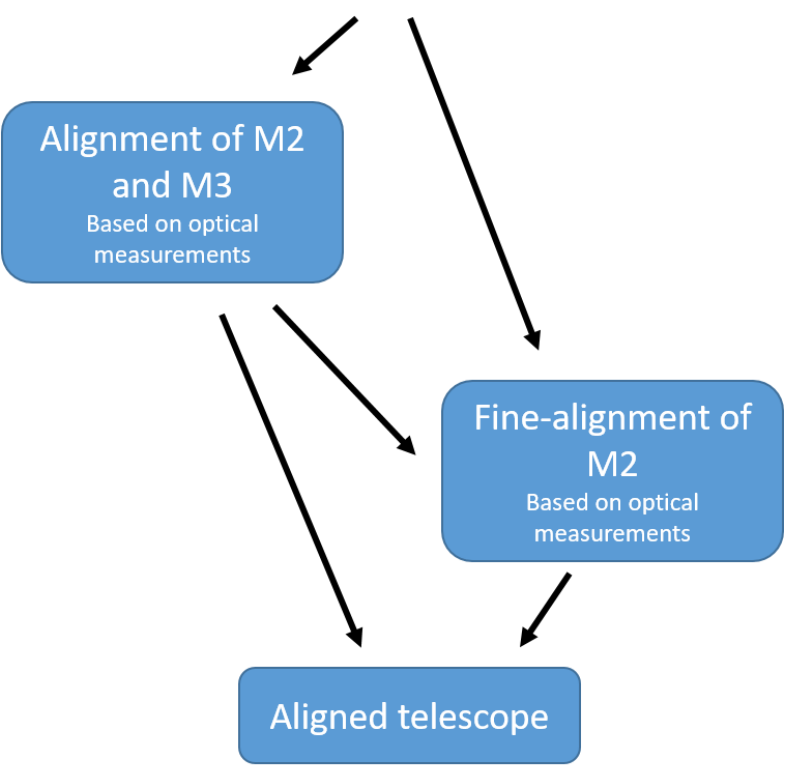

Figure 5 Alignment flow.

The alignment step is followed by a second measurement of the FCI-TA WFE and LOS. The subsequent analysis supported by the SOFA software generates an overview over the current alignment status. The results can be sorted into three categories: 1 . The current WFE and LOS already fulfill the success criteria. The alignment is finished in this case. 2 . The WFE and LOS can be aligned by only fine-movements of M2. In this case, the alignment process continues with the finealignment step. 3. M2 and M3 movements are still required to achieve the WFE and LOS success criteria. In this case, the alignment step is repeated.

In the nominal sequence, the alignment step is followed by the fine-alignment step. Here, a set of precisely manufactured flat shims is used to fine-align the tip/tilt and height of the M2-REM assembly by exchanging the already integrated nominal flat shims with shims of a different thickness. The in-plane degrees of freedom are again controlled by delta movements applied with the push-pull GSE. The delta movements and the required flat shims are calculated by the IFG software based on input generated by the SOFA software.

The fine-alignment step is followed by an optical WFE and LOS measurement. In case the WFE and LOS fulfill the success criteria, the alignment is finished. Otherwise, the fine-alignment can be repeated until the success criteria are fulfilled

The alignment approach was verified by Zemax based Monte-Carlo simulations before application on flight hardware. 


\subsection{Application of concept on first two flight models}

The first two flight models of the FCI-TA have been already aligned and tested. The models are called FM2 and FM3. This subsection summarizes the results of the alignment and draws conclusions with respect to the alignment concept. Table 3 summarizes the WFE and LOS error of the two telescope for every applied alignment step.

The FM2 telescope showed a WFE of $907 \mathrm{~nm}$ (worst-case imagette) and a LOS error of 98 arcsec after the initial placement of the mirrors. Analysis supported by the SOFA software showed that WFE and LOS can be sufficiently corrected by movements of M2 in all degrees of freedom and tip/tilt movements of M3. New wedged shims were produced, in order to apply the movements. The WFE and LOS error after alignment were $63 \mathrm{~nm}$ and $10 \operatorname{arcsec}$, respectively. The measurements do not include a possible alignment of the defocus error by the REM. These numbers already satisfied the success criteria of $102 \mathrm{~nm}$ and 50 arcsec. Therefore, the final fine-alignment step was skipped.

The FM3 telescope showed a WFE of $404 \mathrm{~nm}$ (worst-case imagette) and a LOS error of 67 arcsec after the initial placement of the mirrors. The subsequent analysis showed that the alignment step can be skipped as only movements of M2 (flat shim exchange and push-pull movements in shim plane) were sufficient to fulfill the success criteria for the alignment. In the end, the fine-alignment was performed two times, in order to reach the final WFE and LOS error of $90 \mathrm{~nm}$ and 38 arcsec, respectively, which fulfills the FM3 success criteria of $97 \mathrm{~nm}$ and 50 arcsec.

Table 2 Alignment results.

\begin{tabular}{|l|ll|ll|lc|}
\hline MODEL & \multicolumn{2}{|l|}{ PLACEMENT } & ALIGMENT & \multicolumn{2}{l|}{ FINE-ALIGNMENT } \\
\hline & WFE & Los Error & WFE & Los Error & WFE & Los Error \\
\hline FM2 & $907 \mathrm{~nm}$ & $98 \operatorname{arcsec}$ & $63 \mathrm{~nm}$ & $10 \operatorname{arcsec}$ & - & - \\
FM3 & $404 \mathrm{~nm}$ & $67 \operatorname{arcsec}$ & - & - & $90 \mathrm{~nm}$ & $38 \operatorname{arcsec}$ \\
\hline
\end{tabular}

The alignment concept was successfully demonstrated at two flight models. In both alignment campaigns, it was possible to reach the alignment success criteria while even skipping one alignment step. The concept is proven stable and time efficient, as only a minimal number of correction steps needed to be applied due to the accurate first placement and the accuracy of the alignment steps.

\section{TA OPTICAL PERFORMANCE TESTS AT TELESCOPE LEVEL}

With this section we are going to present the optical performance test results performed on the first TA flight model assembled and delivered by OHB. It was referred in the previous section as FM2 model. After the alignment activity, the TA underwent a series of performance tests, in order to define and validate its main optical requirements and validate simulation models. Those tests were performed in two different stages: a first with the TA in the same configuration as in the alignment, see Figure 3 (called at OBA level), a second, with the OBA mounted onto the interface plate to the satellite (called at TA level). All these measurements were performed at ambient conditions (air at $22^{\circ} \mathrm{C}$ ). Additional to those basic tests, a straylight test and an Opto-Thermo Mechanical (OTM) test were performed, in order to improve correlation of the simulation models with the flight model.

\subsection{Wavefront error and Line of Sight measurements at OBA level}

WFE and LOS are both measured using a Shack-Hartmann Sensor (SHS) positioned at the Telescope focal plane, in the same configuration used for the alignment. This test is basically the same test performed for alignment validation, but after the TA was subject to a vibration test (performed with the purpose to release any stress left in the interfaces) and afterwards optimized for defocus. 

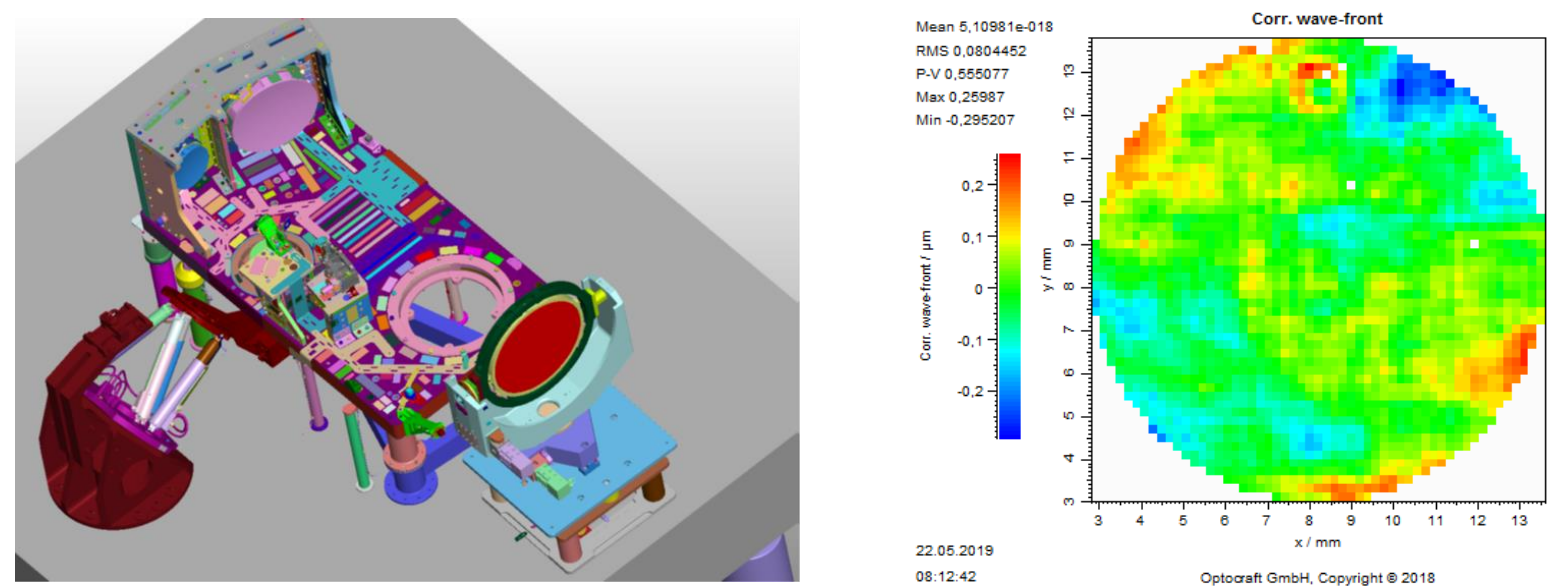

Figure 6 Left - OBA measurement configuration. Right - example of WFE map for center FOV

The SHS was moved to five predetermined positions corresponding to 5 specific FOVs, one in the middle and four at the corners of the Telescope FOV, and used in double pass configuration. The double pass configuration was achieved with the help of a mirror (RMA) placed at the entrance pupil of the telescope. The measurements of the angle of the RMA with respect to the OBA were used to determine the as-built LOS. For FM2, the required WFE after alignment and vibration had to be lower than $102 \mathrm{~nm}$ RMS in the whole FOV, measured with an accuracy of $\pm 15 \mathrm{~nm}$. LOS had no success criterion since its final validation included effects defined only by analysis. Measurement accuracy was of 20 arcsec. The configuration of the measurement is shown in Figure 6, along with an example of WFE measurement for central FOV.

Measurement results are summarized in the following table and show compliance of RMS WFE with the test success criteria. The total RMS is shown below in the table. The WFE results show some residual astigmatism components and a marginal trefoil, even though, as can be seen from WFE in Figure 6, not predominant.

Table 3 WFE and LOS measurements results.

\begin{tabular}{|l|c|c|c|c|}
\hline FOV & $\begin{array}{l}\text { Single Pass Absolute } \\
\text { RMS WFE }(\mathrm{nm})\end{array}$ & Spec. [nm RMS] & $\begin{array}{l}\text { LOS X error } \\
\text { [arcsec] }\end{array}$ & $\begin{array}{l}\text { LOS Y error } \\
{[\operatorname{arcsec}]}\end{array}$ \\
\hline FOV 1 & 40 & \multirow{4}{*}{102} & 7.8 & 0.1 \\
\hline FOV 2 & 59 & & 9.1 & -0.8 \\
\hline FOV 3 & 63 & & 9.3 & -3.0 \\
\hline FOV 4 & 49 & & 7.6 & 1.3 \\
\hline FOV 5 & 55 & & 7.5 & 1.0 \\
\hline
\end{tabular}

The focal plane curvature was additionally verified by the data collected during the above described measurements. From the information of the defocus component, we derived the position of the optimum focus for each field of view with respect to a common plane. The outcome was a curvature of $4 \pm 7 \mu \mathrm{m}$, compliant to a requirement of $\pm 10 \mu \mathrm{m}$.

\subsection{Focal length measurements at OBA level}

In order to verify the paraxial focal length at the central field of view, a specific measurement procedure was planned, using the same equipment as for the WFE measurements. The SHS was moved around the central FOV in small steps of $0.2 \mathrm{~mm}$ in the $\mathrm{FP}\left(0.007^{\circ}\right.$ in FOV domain $)$ in order to recreate a cross, as in the following figure, and verify the focal length along $\mathrm{E} / \mathrm{W}$ and $\mathrm{N} / \mathrm{S}$ directions. 


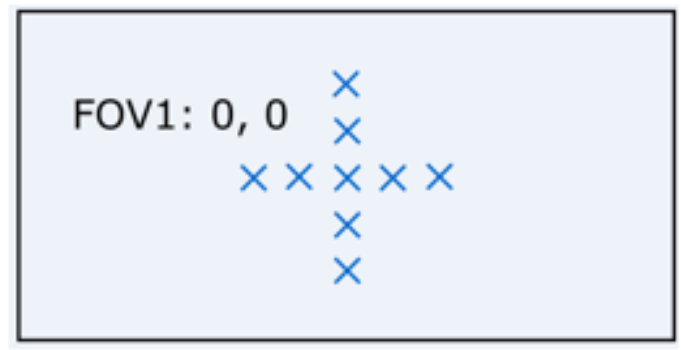

Figure 7 Field angle configurations for measuring of the focal length in the center field point.

The SHS was set to these pre-defined positions in the FP and the RMA was adjusted to minimize the tilt component of WFE. From collected lateral positions of the SHS, $d$, and the angles of the RMA, $\theta$, the focal length $f$ was calculated using a linear regression fit according to the following law:

$$
\tan \varphi=\frac{d}{f}
$$

The required focal length had to be within a range of $\pm 1 \%$ with respect to the design value of $1650 \mathrm{~mm}$. The uncertainty on wavefront sensor position deltas is $5 \mu \mathrm{m}$ while that on RMA's angle is 3 arcsec. Figure 8 shows an example of the E/W focal length data analysis.
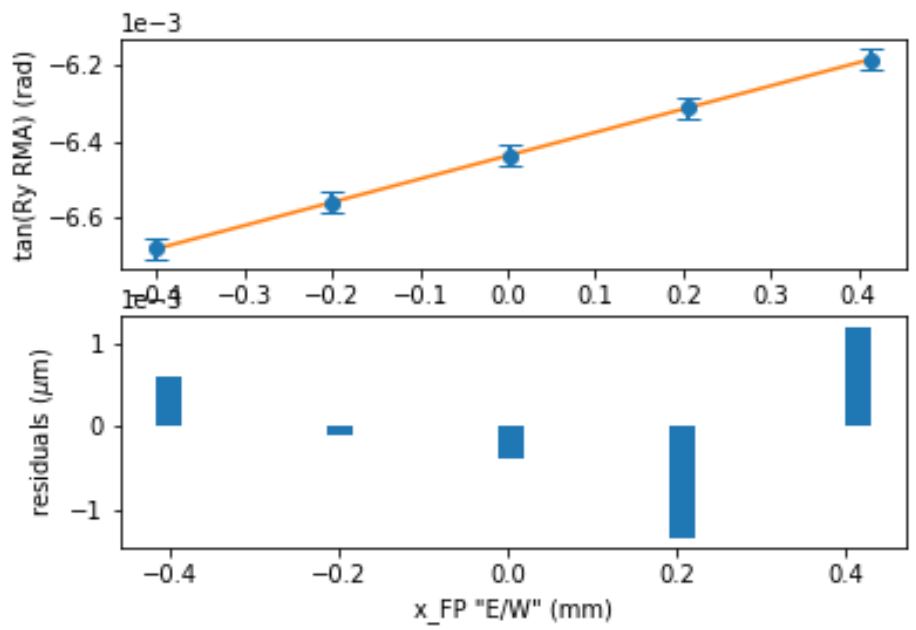

Figure 8 Linear regression of measured data in E/W direction

The results of the analysis, which are compliant with the requirement, are shown in the following table.

Table 4 Focal length measurement results.

\begin{tabular}{|l|c|c|c|}
\hline Axis & $\begin{array}{l}\text { Measured focal } \\
\text { length }[\mathrm{mm}]\end{array}$ & $\begin{array}{l}\text { Accuracy on calculated } \\
\text { value } 2 \sigma[ \pm \mathrm{mm}]\end{array}$ & \multirow{2}{*}{ Spec. [mm] } \\
\cline { 1 - 3 } E/W & 1647 & 9 & \multirow{2}{*}{$1650 \pm 16$} \\
\hline N/S & 1659 & 1 & \\
\hline
\end{tabular}

\subsection{Image rotation measurement at OBA level}

Using the data collected with the SHS and the RMA during the WFE and LOS test described before, it was possible to verify an additional requirement of the TA: the image onto the FP has to maintain a parallelism with respect to the N/S and E/W directions. The SHS was moved to pre-determined positions and the RMA angle was measured. From the requirement $(0.25 \mathrm{deg})$ and the measurement accuracy $( \pm 0.12 \mathrm{deg})$ the measurement had the following condition as success 
criteria: $\varphi \leq 0.13 \mathrm{deg}=(0.25-0.12) \mathrm{deg}$. The following figure gives a figurative impression of the movement of the FOVs associated with the FP positons with respect to the design conditions.

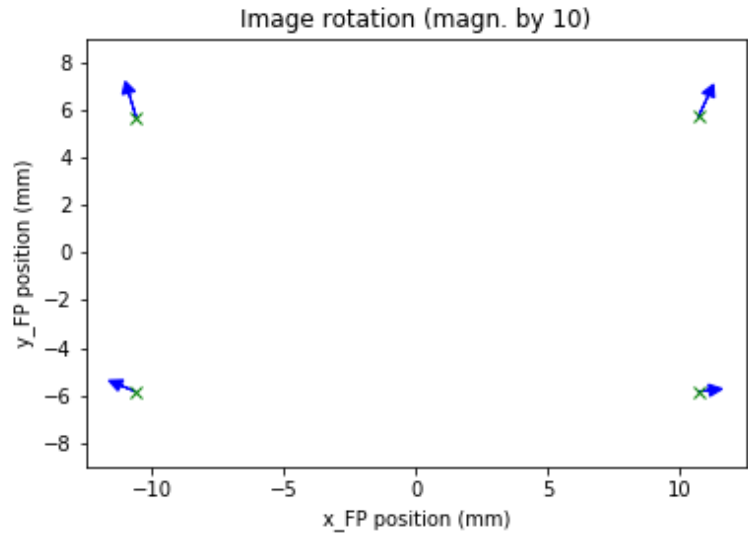

Figure 9 FM2 migration of corners FOVs with respect to design (movements magnified to be better seen).

The calculated rotation had the following result: $\varphi=0.13 \pm 0.12 \mathrm{deg}$. Taking into account the effect of distortion that couldn't be separated from this measurement, the test resulted compliant.

\subsection{Line of Sight at TA level}

As described in section 4.1, LOS was measured alongside WFE but was not supposed to be verified by test at OBA level, but by analysis after the final integration stage, at TA level. After OBA level measurements, the assembly was mounted onto the FCI mounting interface and the scan mirror was integrated. Ultimately, baffles were integrated. The LOS of the TA to be used for requirement verification was the angle of the RMA measured at the central FOV, with the scan mirror at a reference position, corresponding to Nadir pointing in space. The LOS error with respect to the design Nadir pointing position was the first input for requirement verification.

The requirement asks that the Telescope LOS in-flight for the center of the FOV, shall be normal to the FCI mounting interface with an accuracy better than $\pm 0.05^{\circ}=180^{\prime \prime}$.

The measurement performed at the last phase of integration gave -23" of LOS error around X axis and 10" around $\mathrm{Y}$ axis. Measurement accuracy is in the order of 19". The final in-orbit performance was estimated using predictions for contributors to final LOS error. Table 5 lists all these contributors and the final performance with linear summation as a worst case. The performance is compliant with the requirement.

Table 5 Final in-orbit LOS requirement assessment.

\begin{tabular}{|c|c|c|}
\hline in-orbit LOS & Rx [arcsec] & Ry [arcsec] \\
\hline Measurement & -23 & 10 \\
\hline Measurement accuracy & -19 & 19 \\
\hline Gravity & 11 & -12 \\
\hline Thermo elastic & 19 & 12 \\
\hline Moisture Release & 6 & 8 \\
\hline Settling & -10 & -10 \\
\hline Interface distortion & 6 & 12 \\
\hline Linear sum & $\mathbf{- 4 8}$ & $\mathbf{3 9}$ \\
\hline Req. & \pm 180 & \pm 180 \\
\hline
\end{tabular}




\subsection{Straylight Test at telescope level}

The FCI satellite operates at a geostationary orbit. During its lifetime there is a condition, in which the Sun directly illuminates the interior of the telescope; the scan mirror and M1 in particular. This generates straylight, which needs to be carefully analyzed. The Straylight Test performed at telescope level was a measurement aimed at validating the scattering and ghost modeling of mirrors and structures of the TA.

The measurement concept foresees to illuminate the full telescope with a collimated light beam at two different wavelengths. The TA was mounted on a rotating platform and illuminated at varying angles of the LOS with respect to the illuminating source. This was realized using the scan mirror, M0, both in N/S and E/W directions, as well as the rotating platform, which supported the instrument. The angles chosen resulted in the following angle between the LoS and the collimator axis: $1^{\circ}, 3^{\circ}, 5^{\circ}, 9^{\circ}, 15.5^{\circ}$ and $18^{\circ}$, limit being the cut-off angle of the baffle. Wavelengths used to carry on the measurement were $565 \mathrm{~nm}$ and $880 \mathrm{~nm}$; these two wavelengths were chosen because in the visible the scattering effect is more intense and the accuracy of the measurement could be better.

The camera used during the test had a FOV of $1^{\circ} \times 1^{\circ}$ with $1024 \times 1024$ pixels and a pixel pitch of $24 \mu \mathrm{m}$; images, for comparison with test predictions, were cropped to match FCI FOV. For each configuration (angle and wavelength), the intensity count of each pixel was recorded providing, as output, the relative straylight irradiance. As a reference for subsequent measurements, we used the relative irradiance of a direct illumination at a low integration time and low light intensity. Following data were then used as input of a post-process analysis:

- Irradiance of the light source of the entrance pupil: $P_{i}$.

- Integration time of the sensor: $t_{i}$.

- Camera pixel count recorded as a tiff image: $a_{i}$.

For all tiff images we calculated a mean pixel count $\overline{a_{\imath}}$ by dividing the total image count over the pixel number of the corresponding FCI-TA focal area. Therefore, we calculated a straylight weighted pixel count:

$$
C_{\text {stray }}^{i}=\frac{\overline{a_{l}}}{P_{i} t_{i}}
$$

With the first measurement used for calibration with $\mathrm{i}=0$.

We introduced the ratio between the EP and the FP surfaces, in order to compare it to the sun straylight requirement. Using also the trasmissivity and a geometry factor, $S_{x}$, we derived the measured ratio of irradiances:

$$
\frac{E_{\text {straymeas }}}{E_{\text {sourcemeas }}}=\frac{C_{\text {stray }}^{i}}{C_{\text {direct }}^{0}} T(\lambda) \frac{S_{\text {EnP }}}{S_{\text {focal }}}
$$

The method of using the full nominal light image for the determination of the nominal light irradiance response at the detector, bears the risk of calculating the noise over a multitude of pixels into the nominal signal. Therefore, we revised the described method- using a pixel windowing; the amount chosen was of $20 \times 30$ pixels, a bit wider then what needed to be safe with respect to possible change of signal position.

Success criteria for the test were first influenced by some of the limits of the measurements. Data points for angles above $9^{\circ}$ were discarded due to a very low SNR caused by a too low integration time and the data at $1^{\circ}$ resulted compromised by mechanical scatter not simulated in the predictions, hence discarded as well. The success criterion consequently was such that, for the angles between $3^{\circ}$ and $9^{\circ}$ the difference between measurements and predictions had to be lower than the sum of measurement and prediction accuracies. Measurement accuracies were budgeted to be at the level of $64 \%$ and they consist of some fixed uncertainties depending on TA, count errors and dark image subtraction; prediction accuracies were evaluated to be in the order of $150 \%$., They depend on contamination levels estimation and modeling with the software ASAP. The Bidirectional Scattering Distribution Function (BSDF) used for the simulations has been scaled using telescope mirrors contamination, assessed by OHB with witness samples.

An example of the measured images is shown in the following figure. 


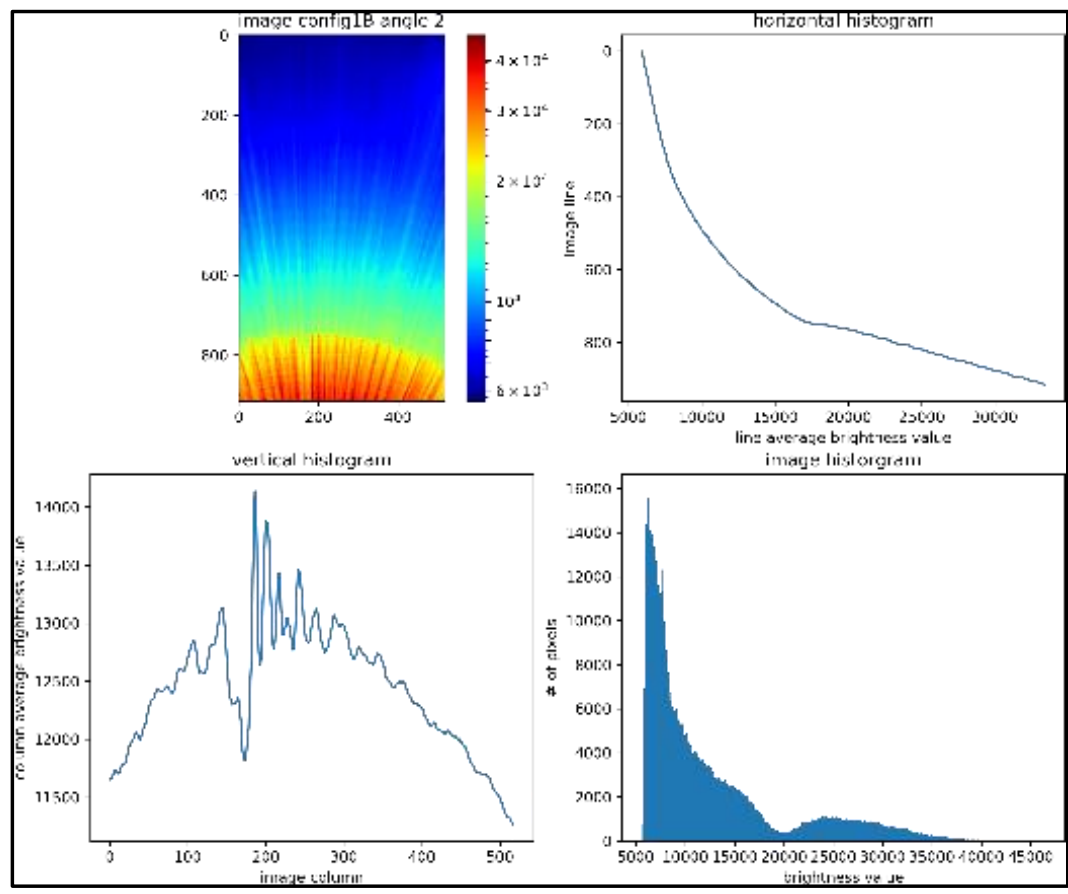

Figure 10 Example of a measurement output. Straylight image at $565 \mathrm{~nm}$ for $1^{\circ}$ angle

The processed values were eventually summarized in a plot for each wavelength. They show the relative irradiance curves for both measurements and predictions (see Figure 11). The measurement error is represented by a straight bar across the value, while the prediction error is represented by a shaded color. We can notice that for each wavelength and limited to the accepted angle values in the success criteria, the measurement and prediction lie within the sum of uncertainties. The test is compliant. Therefore, the BRDF model is validated and it can be used for stray-light correction.
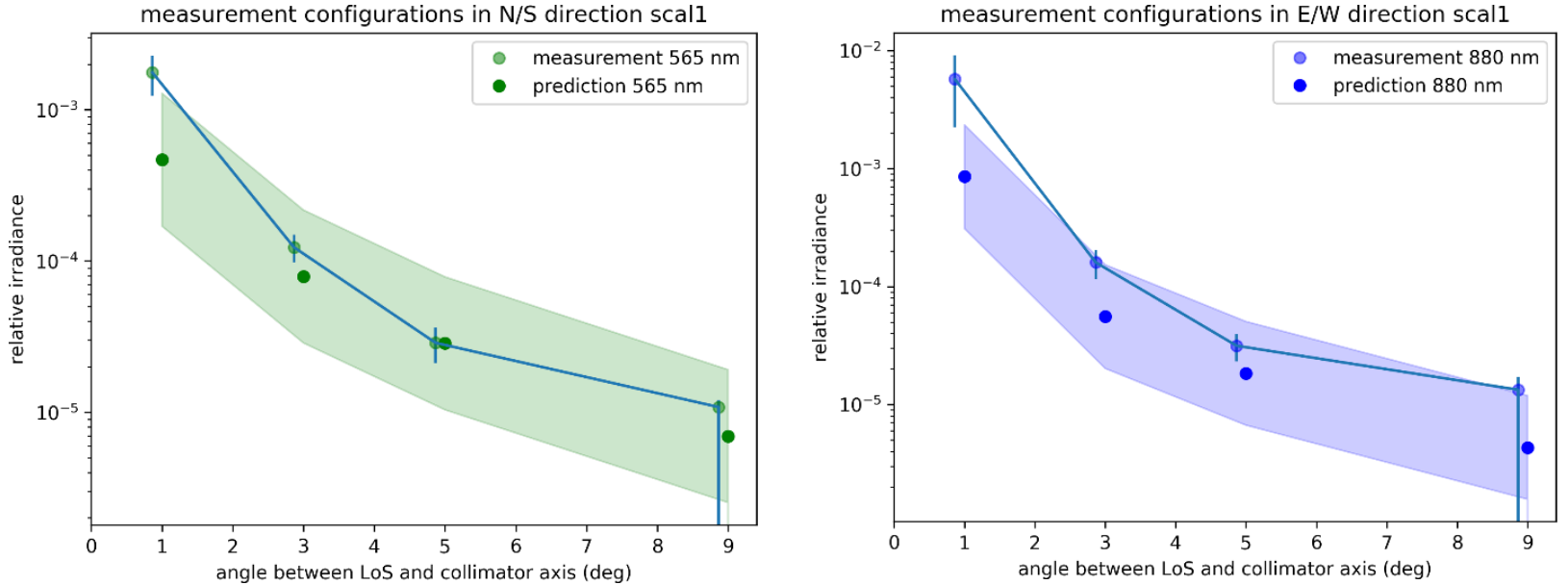

Figure 11 FCI-TA straylight test results for PFM flight model.

\subsection{OTM \& Coefficient of Moisture Expansion (CME) Test}

The OTM and CME test has been conducted in the TAS-F ThermoVacuum chamber over two weeks of testing. The main test objective was to demonstrate that the optical performance parameters, in particular instrument WFE and LoS, could be adequately predicted for in-flight conditions by the available Opto-Thermo-Mechanical analysis models. Thus, the requirement for test was to predict OTM WFE/LoS measured data within $\pm 20 \%$, respectively using the measurement results for model correlation in case of larger deviations. In addition, the test had to demonstrate that the optical WFE 
degradation caused by moisture release, could be compensated by the REM as predicted. The optical performance prediction of the FCI-TA is based on in-orbit temperature prediction via successively: ESATAN (thermal mapping tool into FEM) prediction of related thermo-elastic (or hygro-elastic for CME) deformations and ZEMAX or sensitivity matrices calculation of WFE and LoS performance.

Hence, different temperature scenarios were sequentially generated during the test by the available operational and survival heaters on the most sensitive opto-mechanical components. WFE and LoS were regularly measured each minute via ShackHartmann-Sensor located in a measurement tool called OTM-Tool, mounted on the optical bench. The optical performance were derived based on a double path measurement with one wavelength send out by the OTM-Tool through the telescope and reflected back by the RMA. The OTM-Tool provided an additional $2^{\text {nd }}$ wavelength beam, which was reflected at the end of the OTM-Tool optical path in order to allow for correction of WFE/LoS degradation self-generated by the measurement tool. The RMA and additional Corner Cube Reflectors (CCR) on the instrument were further monitored by a LoS tool called "Cactus", located behind the RMA. With this approach the measured instrument LoS could be cleared from the rigid body movements induced by the test setup, using linear subtraction of the RMA to LoS-Tool and LoS-Tool to CCR measured LoS.
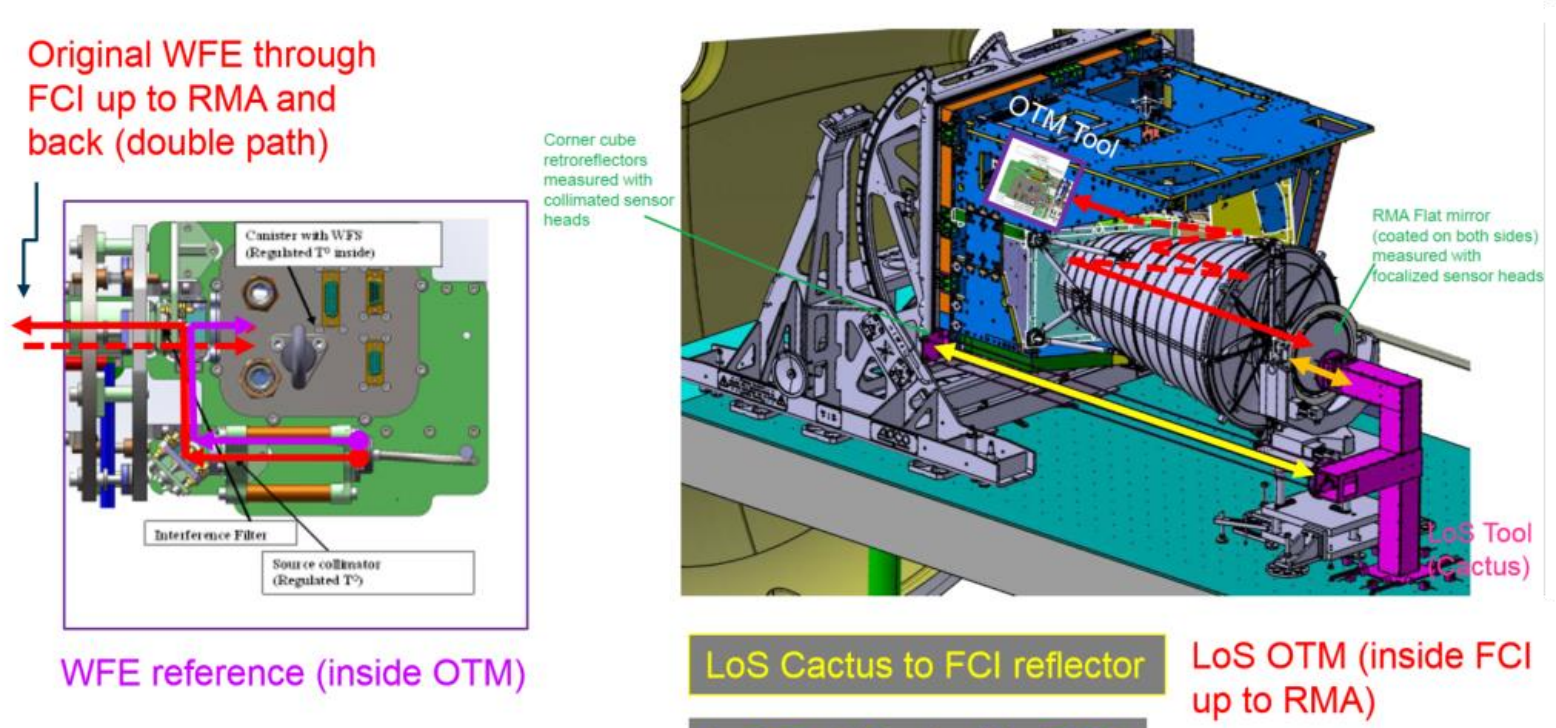

LoS Tool "Cactus" to RMA

Figure 12 OTM \& CME Test setup in TV-Chamber with double path measurement and correlation tools.

In order to distinguish between deformations caused by CME and TED, an outgassing phase (Phase B) has been carried on after pump down and initial temperature stabilization to $20^{\circ} \mathrm{C}$ (Phase A). The system was maintained for around 3 days at $30^{\circ} \mathrm{C}$ until the measured WFE/LOS change became one order of magnitude lower than expected by the upcoming TED scenarios. Then we started the core TED phase (Phase C). Ten different temperature scenarios have been generated by sequential heat up of the subsystems and Optical Bench, from initially stabilized $20^{\circ} \mathrm{C}$ by $+10 \mathrm{~K}$ until all units reached a homogeneous and stable temperature of $30^{\circ} \mathrm{C}$. The $\mathrm{CME}$ evolution has been then again monitored in Phase $\mathrm{D}$ and at the end of Phase E to allow for better CME evaluation and extrapolation. The sequence of test phases is shown in the following figure. 


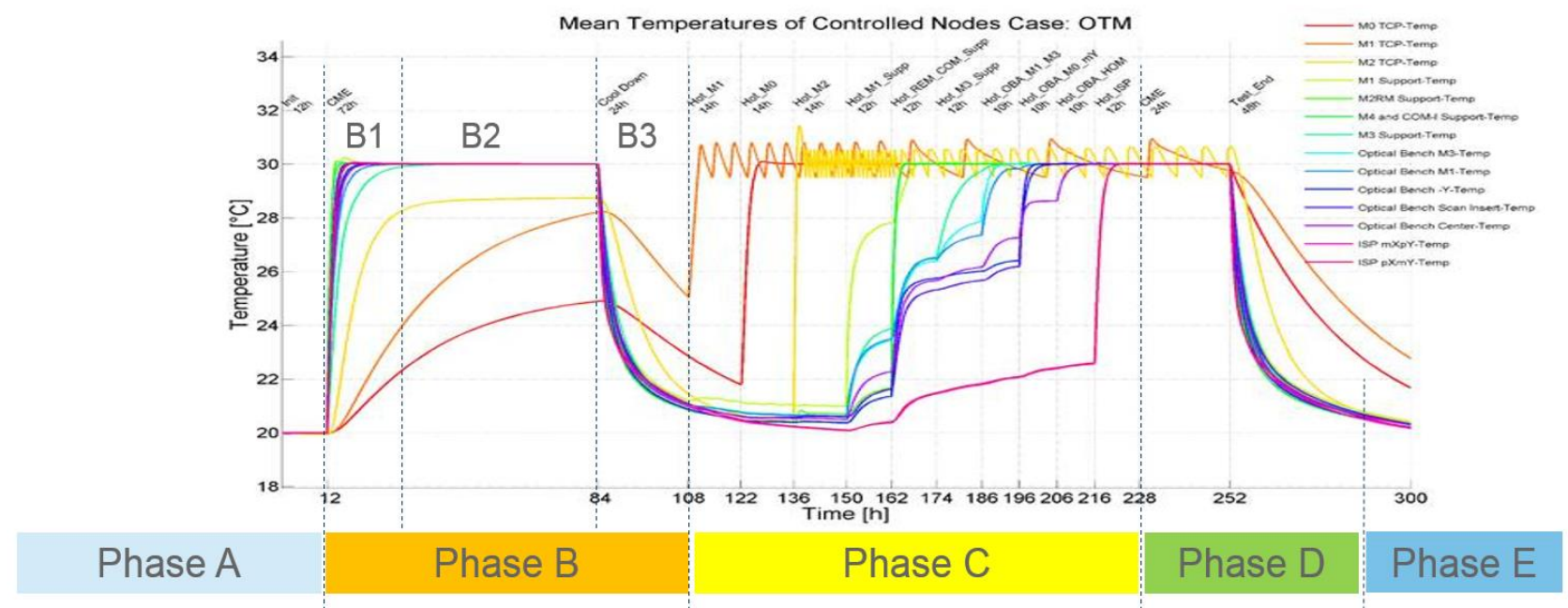

Figure 13 OTM \& CME Test Phases in TV chamber.

The initial WFE was around $90 \mathrm{~nm}$ after pump down and stabilization of the chamber environment to $20^{\circ} \mathrm{C}$ with an optimized defocus to nearly $0 \mathrm{~nm}$ using the REM. After Phase $\mathrm{B}$ and stabilization to $20^{\circ} \mathrm{C}$ again, the defocus term degraded by around $-80 \mathrm{~nm}$ due to moisture release; total WFE changed to $120 \mathrm{~nm}$. Based on test evaluation, around $45 \%$ of the total moisture was released up to the begin of Phase C, i.e. the total expected change in WFE caused by CME shrinkage would be up to $175 \mathrm{~nm}$ in defocus only. However, the FCI has been intentionally designed so that higher order terms of the wavefront are almost not affected by structural deformations caused by either temperature variation or moisture release. Hence, the CME WFE degradation could be fully compensated by the REM down to again $90 \mathrm{~nm}$ at the beginning of Phase C.

The heating of all major optical components by a delta temperature of $+10 \mathrm{~K}$ in Phase $\mathrm{C}$ increased the total WFE up to $340 \mathrm{~nm}$, whilst the LoS increased in total by 20 arcsec. The total WFE change was again primary driven by the defocus term of $-320 \mathrm{~nm}$, as predicted. Figure 14 illustrates the measured versus predicted evolution of the major performance parameters after model correlation.

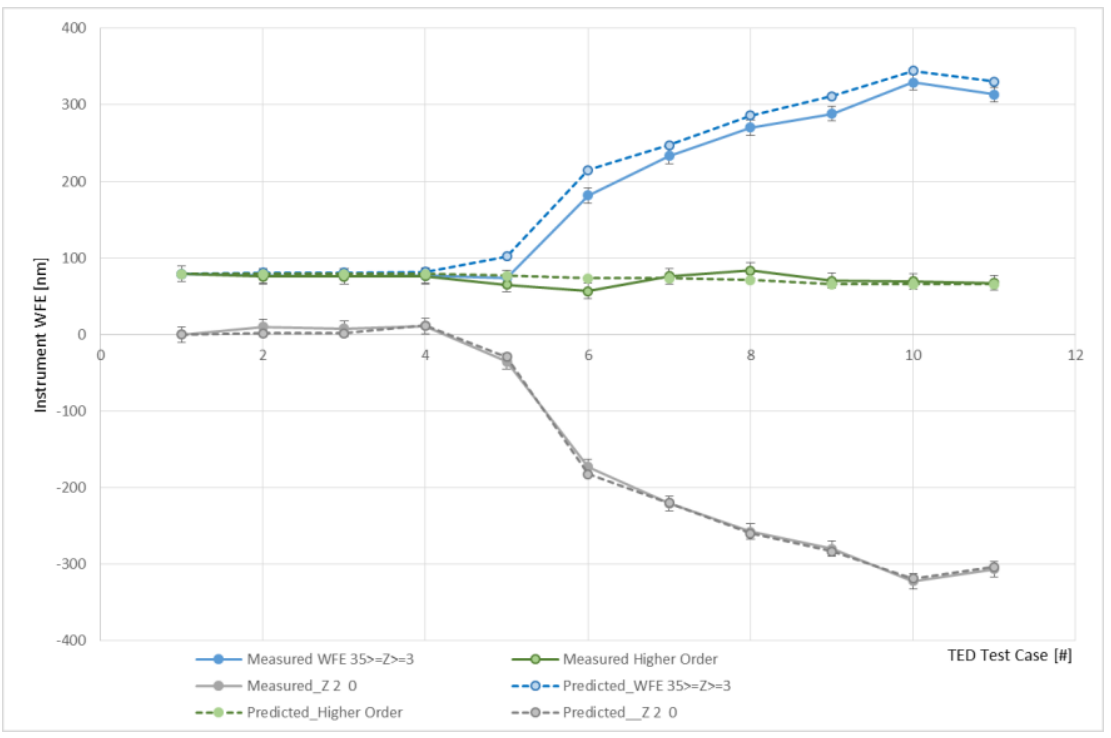




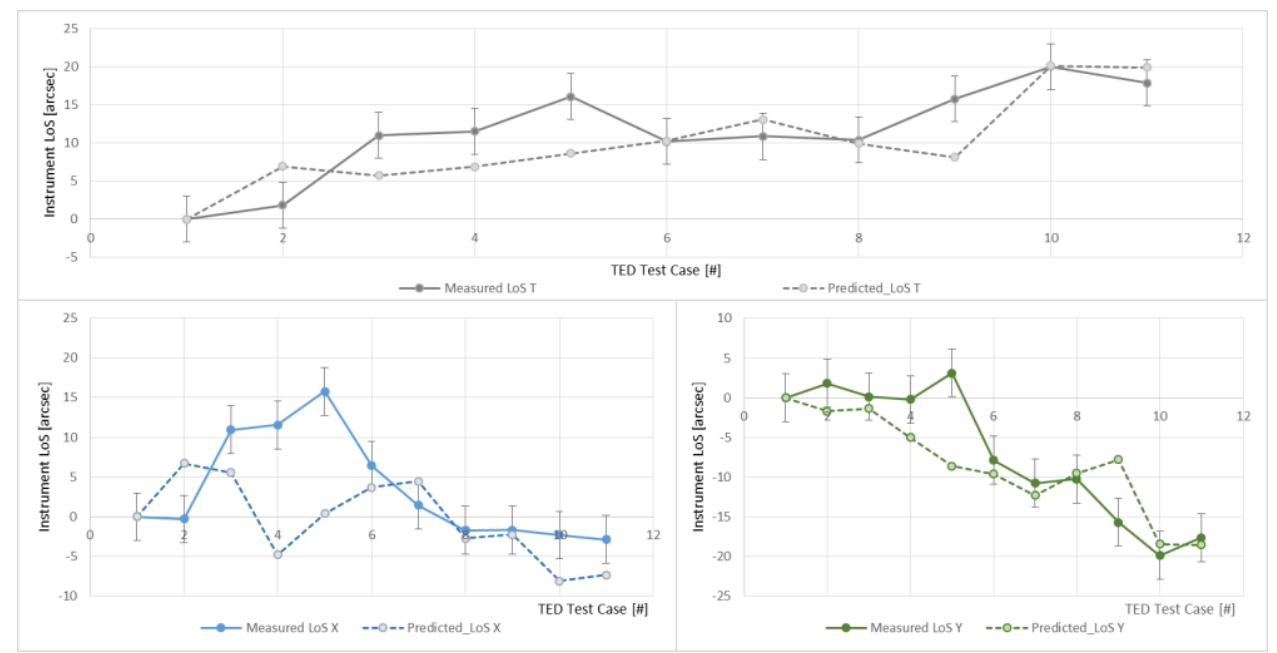

Figure 14 Measured versus Predicted instrument WFE and LoS for tested TED scenarios [\#] after model correlation. WFE measurement accuracy is $\pm 10 \mathrm{~nm}$. LoS measurement accuracy is \pm 3 arcsec.

It should be noted the WFE has been underestimated by around $-45 \%$ prior to the OTM test correlation, whilst LoS change had a prediction failure up to factor 7 in worst case. Here it is noteworthy to mention that the STOP model had already been initially correlated by the results obtained from a Video-Grammetry-Test (VGT) done on the FCI STM, where the WFE was overestimated by around $+20 \%$ with regards to the predictions using the materials theoretical data. It can thus be concluded, the model had been correlated in the false direction prior, probably thanks to insufficient measurement accuracy of the VGT. Hence, the CFRP-Honeycomb CTE and shear modulus just needed to be slightly adjusted with regards to the theoretical material properties to fit the OTM measured results well within the requirement. The excellent WFE prediction after final correlation is shown in the left plot in Figure 14.

After WFE correlation, the largest remaining LoS prediction failure was found for heating the SCAN mirror M0 and the COM/M4 support on the OBA. However, no model correlation approach could be found which increased prediction confidence in those scenarios within the anticipated $\pm 20 \%$ requirement, without worsening WFE or LoS prediction for other tested scenarios. Hence, a higher STOP analysis uncertainty factor of safety in range of 2-3 has been taken into account on top of the predicted in-orbit LoS RX and RY performance.

The main root cause of the prediction uncertainty of LoS and WFE is expected to be found by the fact that total WFE is driven by defocus mainly, which primary results from the global expansion and shrinkage of the optical bench. This global deformations can be well predicted by the STOP model and the WFE is less affected by small local rotations of the mirrors as well as the rotation sign does not matter when Zernikes are summed up by root-sum-square. The LoS instead is highly sensitive to local mirror rotations, especially the flat M0 (scan mirror), and each interface rotation needs to be predicted in all axis with the correct \pm sign in order to compensate or add each optical element contribution to the total instrument LoS correctly. It appears that the available STOP model does not fully represent the TED behavior or the SCAN mirror (M0) rotational pivots and flexural mounting up to the yoke, as well as the FEM modelling of the box shaped COM support does not consider the very large of amount of inserts and panel connections sufficiently and thus cause an LoS prediction with higher uncertainty.

However, even with larger WFE and the additional LoS prediction after correlation, the predicted total FCI in-orbit performance remains within the mission requirements

\section{CONCLUSIONS}

We have presented in this paper the results of the alignment and the performance test of the FCI Telescope Assembly first flight model. The alignment success criteria for FCI-TA were driven mainly by WFE and LOS. OHB developed an integration and alignment procedure, based both on Optical Ground Equipment (OGSE) and software, which proved successful with two successive flight models. At the end of 2020 a third flight model has been aligned with the same procedure; the model confirmed great results in terms of WFE and LOS (as well as the other performance). This procedure is now a standard for future project within the company. 
A series of OGSE let us test the FCI-TA through different steps of its integration. All optical performance described in section 4 were compliant with respect to the success criteria. WFE performance proved in particular stable after successive integration of the scan mirror and the baffles. With the FCI-TA all assembled, we characterized the stray-light on-ground. The test demonstrated a correlation among the flight and the simulated models, making it possible to provide the necessary inputs for in-orbit correction algorithms. The correlation of the structural and thermal model, performed with the OTM \& CME test proved, with some correction, excellent for the WFE prediction. LoS correlation was a bit more problematic but still within mission requirements.

During the first quarter of 2020 the first FCI-TA flight model, FM2, along with the detection assembly, SSDA [1], were integrated together, a TAS premises, to form the FCI PFM. This model will then undergo optical vacuum testing at the beginning of 2021. Since the integration of the first flight model two other TA have been aligned at OHB proving the validity of the design and the alignment procedure.

\section{ACKNOWLEDGMENTS}

This work is performed under ESA contracts to TAS-F and OHB. The authors would like to thank first the instrument prime TAS and ESA for the fruitful support and collaboration which led to this successful instrument. Moreover we would like also to acknowledge the collaboration of every sub-contractor.

\section{REFERENCES}

[1] Ouaknine J. et al, "FCI PFM optical test results", ICSO 2020.

[2] J. Kolmeder, A. Kuisl, B. Sang, M. Lettner, A. Godenir, M. Glier, M. Sornig, S. Fischer, "Optical integration process for the earth-observing satellite mission ENMAP," Proc. SPIE 10562, International Conference on Space Optics — ICSO 2016, 1056226 (25 September 2017); https://doi.org/10.1117/12.2296080. 